\title{
Respiratory rate and body mass in the first three years of life
}

Luigi Gagliardi, Franca Rusconi, and the working party on respiratory rate

\begin{abstract}
The correlation between respiratory rate and body mass in the first three years of life was examined in 635 infants and children, aged between 15 days and 3.5 years. Assessments were made during quiet sleep and when awake and calm. Their body weight (2.8-20.5 kg) was measured at the same time.

Respiratory rate decreased exponentially with increasing body weight. After log transformation of the data, a linear correlation was found between $\log$ (respiratory rate) and $\log$ (body weight) both during the sleeping and the wakeful states. For each body weight group, boys had about a $6.5 \%$ higher respiratory rate than girls, but both the boys' and the girls' lines were parallel. The slope of the line was $\mathbf{- 0 . 4 3}$ during sleep and $\mathbf{- 0 . 4 1}$ during wakefulness, indicating that the respiratory rate: body weight ratio is not a constant in either state, and that it decreases as body weight increases. Adding age to the model did not significantly increase the precision of the fitting.

A simple equation of the form respiratory rate $=\mathbf{a} \cdot \mathbf{b o d y}$ weight $^{\mathrm{b}}$, can accurately describe the decrease in respiratory rate with growth. Smaller (hence younger) infants and children have a higher respiratory rate per unit body weight than larger (hence older) children.

(Arch Dis Child 1997;76:151-154)
\end{abstract}

Keywords: respiratory development; body weight; breathing rate; sleeping state; wakeful state.

Neonatology, Ospedale Niguarda Ca' Granda, Milan, Italy

L Gagliardi

Department of

Paediatrics 2,

University of Milan

and ICP, Milan, Italy

F Rusconi

${ }^{*}$ Members of the working party on respiratory rate included: M Castagneto; G Leo N Porta; S Razon (Milan); A Pellegatta (Busto Arsizio)

Correspondence to: Dr Luigi Gagliardi, Divisione di Patologia Neonatale,

Ospedale Niguarda

$\mathrm{Ca}^{\prime} \mathrm{Granda}$,

Piazza Ospedale Maggiore 3

20162 Milano, Italy.

Accepted

24 September 1996 children, and children less frequently than infants and neonates. ${ }^{10}{ }^{11}$ No data are, however, available on the relation between respiratory rate and body mass during growth. Like many other variables, body mass changes very rapidly in the first years of life. In a previous study we measured respiratory rate in the first three years of life in a large sample of normal subjects in both sleeping and wakeful states, and described its diminution with age. ${ }^{10}$ The aim of the present study, performed on the same sample, was to investigate the relation between respiratory rate and body weight. The influence of other variables such as gender and age on the estimation was also investigated.

\section{Methods}

This was an observational, cross sectional study. Full details of recruitment of the sample have been reported elsewhere. ${ }^{10}$ Briefly, 662 infants and children aged 15 days to 3.5 years were enrolled in the study: 334 were seen in day care centres, and 328 as inpatients or outpatients. The main diagnoses were gastrointestinal and urological disorders, skin diseases, and orthopaedic problems. Exclusion criteria were the presence of any chronic or severe illness, dehydration, or a history of fever or respiratory findings suggesting a respiratory infection in the previous two weeks. No preterm infants were included.

Respiratory rate was assessed by four investigators, each one assessing more than 100 subjects, by listening to breath sounds for 1 minute with a stethoscope. In each child respiratory rate was recorded by the same assessor, both when the child was awake and calm, and when $\mathrm{s} / \mathrm{he}$ was in quiet sleep, according to behavioural criteria (sleep without spontaneous movements, including no eye movement or vocalisation). The repeatability of respiratory rate counting, expressed as standard deviation (SD) of the differences between duplicate counts obtained by the same investigator 30-60 minutes apart, was 1.7 and 2.5 breaths $/ \mathrm{min}$ in data on sleeping and awake infants, respectively, at a mean respiratory rate of 30.1 and 39.7 breaths per minute, respectively. ${ }^{10}$ Body weight was measured using standard scales during medical examination. For the present study, 27 subjects were excluded from the original sample after close scrutiny of the forms, eight because body weight data were missing, and 19 because the reason for seeking medical attention was possibly associated with body weight disturbances, regardless of whether body weight was within $(n=8)$ or outside $(n=11)$ the 5 th-95th National Center for Health Statistics centile range. ${ }^{12}$ Thus 635 records (for 353 boys and 282 girls) were analysed. 
96478.f1

Figure 1 Scattergram of respiratory rate $v$ body weight in sleeping subjects.

$96478 . f 2$

Figure 2 Scattergram of respiratory rate $v$ body weight in awake subjects.

For the statistical analysis, standard least squares linear models were used. ${ }^{13}{ }^{14}$ All calculations were carried out using the Minitab statistical package.

\section{Results}

Body weight increased with postnatal age, ranging from 2.8 to $20.5 \mathrm{~kg}$, and was larger in boys than girls. Body weight was lower than the 5 th centile for age ${ }^{12}$ in $5.7 \%$ of boys, and higher than the 95 th centile in $7.9 \%$; the corresponding figures for girls were $8.2 \%$ and $8.5 \%$, respectively. Mean $\mathrm{Z}$ scores (subject's body weight minus mean body weight for age/SD for age) were 0.004 (SD 1.14) for boys, and 0.078 (DS 1.17) for girls, both not significantly different from zero. These values indicate a slightly larger spread than expected, but no systematic bias in the sample.

The relation between respiratory rate and body weight was curvilinear both in data from wakeful and sleeping infants, and the equation respiratory rate $=\mathrm{a} \bullet$ body weight $^{\mathrm{b}}$ was fitted. When both respiratory rate and body weight are $\log$ transformed, the relation is that of a straight line. $\log$ (respiratory rate) = $\log (a)+b \cdot \log ($ body weight $)$, and $\log (a)$ and $b$ can be estimated with a standard least squares method. Logs to base 10 were used.
The relation between respiratory rate and body weight differed in boys and girls: at each body weight, boys had a higher respiratory rate than girls. The scattergram of respiratory rate in the sleeping state against body weight in boys and girls is shown in fig 1, and the corresponding plot for awake data in fig 2 . Both in sleeping and in wakeful states, the slope of the regression lines of $\log$ (respiratory rate) on log(body weight) was not significantly different in boys and girls (Student's $t$ test $=0.70$ and 0.11 ; $\mathrm{p}>0.48$ and $>0.9$ in data from sleeping and wakeful infants, respectively) - gender did not change the relation between $\log$ (body weight) and $\log$ (respiratory rate), but only influenced the intercept. We fitted parallel regression lines, the distance between the lines being an estimate of the sex difference. ${ }^{14}$

For data on sleeping subjects, a linear regression was sufficiently accurate to describe the relation between $\log$ (respiratory rate) and $\log$ (body weight), and the second degree term was not significant. The estimated parameters of the equation describing the function are shown in table 1 .

Conversely, for data on awake subjects, a slight curvilinear shape was found, and a second degree function was also tried. Although the second degree term was significant, the importance of deviation from linearity in data on awake subjects should not be overemphasised. The difference in the goodness of fit between the linear and quadratic functions was trivial, as shown by the finding that $R^{2}$ was 0.474 with the linear equation and 0.486 with the quadratic equation, that the residual SD was similar in both models (0.0866 with the linear function; 0.0857 with the quadratic function), and that in $96.5 \%$ of the observations the discrepancy in fitted (estimated) values obtained with the two equations was less than two breaths/min. Thus the simpler linear equation was also preferred ${ }^{14}$ for data on awake subjects (table 1 ).

In both the sleeping and wakeful states, the exponent was negative and significantly smaller than 1, indicating that respiratory rate decreases with increasing values of body weight, and that the decrease is not directly proportional to the body weight increase but is simply slower. The effect of gender was quantitatively very similar in both sets of data (table 1): at each body weight, the respiratory rate of boys was $6.6 \%$ higher during sleep, and $6.4 \%$ higher during wakefulness, than that of girls.

A model including age (after log transformation) was also tried, and the estimated parameters are reported in table 2. In this sample, however, $\log$ (age) was highly correlated with $\log$ (body weight): $r=0.96$. As a result, the increase in explained variance $\left(R^{2}\right)$ and the decrease in residual SD were trivial both during sleep and during wakefulness. The inclusion of $\log ($ age $)$ was significant only in data on awake subjects, but in those for sleeping subjects, it rendered non-significant the $\log$ (body weight) term. Using the technique suggested by $\mathrm{Healy}^{13}$ — that is, regressing $\log$ (respiratory rate) on the mean and difference of $\log$ (body weight) and $\log ($ age $)$ instead 
Table 1 Values (SE) of estimated parameters of equations describing log(respiratory rate) as a function of log (body weight) and sex, according to sleeping or waking, (Logs are to base 10)

\begin{tabular}{lllll}
\hline & Asleep subjects & $p$ & Awake subjects & $p$ \\
\hline Log(body weight) & $-0.430(0.018)$ & $<0.001$ & $-0.412(0.017)$ & $<0.001$ \\
Sex & $0.0276(0.00728)$ & $<0.001$ & $0.0268(0.00698)$ & $<0.001$ \\
Constant & $1.830(0.0172)$ & $<0.001$ & $1.923(0.016)$ & $<0.001$ \\
Residual SD & 0.0904 & & 0.0866 & \\
$R^{2}$ & 0.474 & & 0.474 & \\
\hline
\end{tabular}

* Coded $1=$ males; $0=$ females.

Table 2 Values (SE) of estimated parameters of equations describing log(respiratory rate) as a function of log (body weight), log(age) and sex, according to sleeping or waking, (Logs are to base 10)

\begin{tabular}{lllll}
\hline & Asleep subjects & $p$ & Awake subjects & $p$ \\
\hline Log(body & $-0.085(0.059)$ & 0.151 & $-0.132(0.057)$ & 0.022 \\
$\quad$ weight) & $-0.134(0.022)$ & $<0.001$ & $-0.109(0.021)$ & $<0.001$ \\
Log(age) & $-0.016(0.007)$ & 0.025 & $0.018(0.007)$ & 0.012 \\
Sex & $1.641(0.035)$ & $<0.001$ & $1.769(0.034)$ & $<0.001$ \\
Constant & 0.0879 & & 0.0849 & \\
Residual SD & 0.503 & & 0.495 & \\
$R^{2}$ & & & & \\
\hline
\end{tabular}

^ Coded $1=$ males; $0=$ females.

of on the variables themselves, yielded non significant results for the difference, confirming that body weight and age provide essentially the same information.

\section{Discussion}

This study shows that respiratory rate can be expressed as a simple function of body weight during the first three years of life, both in sleeping and wakeful subjects, and that the respiratory rate:body weight ratio in humans decreases with growth. This means that the expression 'respiratory rate per unit body weight' is potentially misleading, and must be used with great caution to avoid false conclusions being drawn. ${ }^{15}$

As in mammals, ${ }^{3}$ a relation between respiratory rate and body size in infants and children might be expected. Although the control of respiratory rate is not completely understood, minute ventilation is known to be intimately related to the metabolic rate; the level of ventilation is regulated so as to match metabolic demands (carbon dioxide production and oxygen consumption), and higher metabolic rates must be accompanied by larger ventilation levels. It has been known for many years that metabolic rate (and hence oxygen consumption per unit body weight, as metabolism is mainly aerobic) is not a constant per unit body weight, but decreases with increasing body weight $^{3}{ }^{16-18}$ - that is, larger animals have a smaller metabolic rate per unit body weight than smaller ones. Thus smaller animals must match higher energy expenditure per unit body weight with higher ventilation levels per unit body weight. An increase in alveolar ventilation can be accomplished by several breathing strategies, including increasing tidal volume and/or respiratory rate, or reducing dead space. It is generally believed that, for a given level of ventilation, each animal spontaneously chooses a combination of tidal volume and respiratory rate that minimises either the work of breathing $^{119}$ or the average force of respiratory muscles. ${ }^{2}$ Mortola has pointed out that in neonates and younger children increasing respiratory rate (instead of increasing tidal volume) is probably a cost effective strategy to cope with higher ventilation levels. ${ }^{7}$ The strategy of changing respiratory rate rather than tidal volume agrees with the data obtained across various species, which show that higher minute ventilation is achieved by higher respiratory rate whereas tidal volume and dead space remain constant per unit body weight. ${ }^{3}$ In resting humans both tidal volume and dead space per body weight remain essentially unchanged from birth to adulthood (about 7 and $2.2 \mathrm{ml} / \mathrm{kg}$ body weight, respectively). ${ }^{20}$

Our study further supports these observations, the major finding being that respiratory rate per unit body weight in neonates, infants, and children is not constant but decreases with increasing body weight. During quiet sleep in the first three years of life respiratory rate varies inversely in proportion to about body weight $^{-0.4}$. According to our equation, a sleeping boy weighing $5 \mathrm{~kg}$ has an expected respiratory rate of 36.1 breaths/min (respiratory rate: body weight ratio $=7.2$ ), compared with 19.9 breaths/min (respiratory rate:body weight ratio $=0.995$ ) in one weighing $20 \mathrm{~kg}$.

Boys, whose metabolism per unit body weight is higher than that of girls, ${ }^{16}{ }^{1721}$ also have a higher respiratory rate for any given body weight than girls (about $6.5 \%$ both during sleep and wakefulness).

Admittedly, this model - and its explanation in biological terms - is rather simplistic. Even with the knowledge of body weight, sex, and age, only about $50 \%$ of the scatter (variance) of respiratory rate counts is explained, and much of it is due to body weight. The residual SD is around 0.09 in $\log$ terms - around $23 \%$ in either direction. We did not measure length, so that it is impossible to know how much this would have changed predictions. The large scatter around predictions, however, is not only due to poor fitting, but to real dispersion of respiratory rate counts ${ }^{10}{ }^{11}$ - that is, biological differences across subjects.

The relation between body weight and respiratory rate should not be dismissed as 'merely descriptive.' The fact that the same very simple basic relation (the allometric equation) which holds for all mammals is also adequate for humans despite variations in age and sex, indicates that the same 'lawlike relationship' is true. ${ }^{22}$

Obviously, our results cannot be extrapolated beyond the range of weights (age) used to construct the curves. Increasing the age range, besides modifying the slope, would probably have introduced a substantial deviation from linearity with resulting inaccuracies in estimation.

These data were presented in part at the annual Congress of the European Respiratory Society, September 1995, Barcelona, Spain.

1 Crosfill ML, Widdicombe JG. Physical characteristics of the chest and lungs in the work of breathing in different mammalian species. F Physiol (Lond) 1961;158:1-14. 
2 Mead J. Control of respiratory frequency. 7 Appl Physiol 1960;15:325-36.

3 Mortola JP. Dynamics of breathing in newborn mammals. Physiol Rev 1984;67:187-243.

4 Guyton AC. Analysis of respiratory patterns in laboratory animals. Am F Physiol 1947;150:79-83.

5 Drorbaugh JE. Pulmonary function in different animals. $f$ Appl Physiol 1960;15:1069-72.

6 Stahl WR. Scaling of respiratory variables in mammals. $f$ Appl Physiol 1967;22:453-60.

7 Mortola JP. Some functional mechanical implications in the structural design of the respiratory system in newborn mammals. Am Rev Respir Dis 1983;128:S69-72.

8 Heusner AA. Mathematical expression of the effects of changes in body size on pulmonary function and structure. Am Rev Respir Dis 1983;128:S72-4.

9 Mortola JP. Breathing patterns in newborns. f Appl Physiol 1984;56:1533-40.

10 Rusconi F, Castagneto M, Gagliardi L, Leo G, Pellegatta A, Porta N, et al. Reference values for respiratory rate in the first 3 years of life. Pediatrics 1994;94:350-5.

11 Marks MK, South M, Carlin JB. Reference ranges for respiratory rate measured by thermistry (12-84 months). Arch Dis Child 1983;69:569-72.
12 Hamill PVV, Drizd TA, Johnson CL Reed RB, Roche AF, Moore WM. Physical growth: National Center for Health Moore WM. Physical growth: National Center for He
Statistics percentiles. Am f Clin Nutr 1979;32:607-29.

Statistics percentiles. Am f Clin Nutr 1979;32:607-29.
13 Healy MJR. Multiple regression (1). Arch Dis Child 1995;73:177-81.

14 Healy MJR. Multiple regression (2). Arch Dis Child 1995; 73: $270-4$.

15 Cooper DM, Berman N. Ratios and regressions in body size and function: a commentary. F Appl Physiol 1994;77:2015-7. 16 Talbot FB. Basal metabolism of children. Physiol Rev 1925; 5:477-517.

17 Kleiber M. Body size and metabolic rate. Physiol Rev 1947; 27:511-41.

18 Holliday MA. Body composition and energy needs during growth. In: Falkner F, Tanner JM, eds. Human growth. 2 Postnatal growth. London: Baillière-Tindall, 1978:117-39.

19 Otis AB, Fenn WO, Rahn H. Mechanics of breathing in man. F Appl Physiol 1950;2:592-607.

20 Polgar G, Weng TR. The functional development of the respiratory system. From the period of gestation to adulthood.
polgar $\mathrm{G}$. Weng piratory system. From the period of gesta

21 Schofield C. Predicting basal metabolic rate, new standards and review of previous work. Hum Nutr Clin Nutr 1985;39 (suppl 1):5-42.

22 Ehrenberg ASC. The elements of lawlike relationships. $\mathcal{f}$ Roy Statist Soc A 1968;131:280-302.

\section{Fetal heart rate monitoring}

It is estimated that some 10 to $20 \%$ of cerebral palsy originates during labour, $70 \%$ before the onset of labour, and $10 \%$ after birth. At best, therefore, fetal heart rate monitoring (FHRM) could influence $20 \%$ of cerebral palsy. Work in California has shown the probable influence to be much less (Karin B Nelson and colleagues, New England fournal of Medicine 1996;334: 613-8).

The FHRM records of 395 children were reviewed. All were singletons born at term weighing $2500 \mathrm{~g}$ or more and 95 had moderate or severe cerebral palsy at the age of 3. Multiple late decelerations were associated with a fourfold, and decreased beat-to-beat variability with a threefold, increase in risk of cerebral palsy. Despite that, only two in 1000 children showing the FHRM abnormalities developed cerebral palsy if they survived to age three. (These data give no information about fetal death, early neonatal death, or death before age 3.)

Other evidence suggests that FHRM has not reduced rates of cerebral palsy and these authors argue that performing caesarean section because of these abnormalities on monitoring could potentially be responsible for an increase in maternal morbidity with no commensurate benefit to the fetus.

ARCHIVIST 